\title{
Peroxisome proliferator-activated receptor delta facilitates lipid secretion and catabolism of fatty acids in dairy goat mammary epithelial cells
}

\author{
H. B. Shi, ${ }^{* 1}$ C. H. Zhang, $\dagger^{1}$ W. Zhao, $\dagger$ J. Luo, $t^{2}$ and J. J. Loor $\ddagger^{2}$ \\ *Key Laboratory of Silkworm Bioreactor and Biomedicine of Zhejiang Province, College of Life Science, Zhejiang Sci-Tech University, Hangzhou, \\ Zhejiang Province 310018, China \\ †College of Animal Science and Technology, Northwest A\&F University, Yangling, Shaanxi, 712100, P.R. China \\ ¥Mammalian NutriPhysioGenomics, Department of Animal Sciences and Division of Nutritional Sciences, University of Illinois, Urbana 61801
}

\section{ABSTRACT}

In rodents, peroxisome proliferator-activated receptor delta (PPARD) is associated primarily with catabolism of fatty acids. However, the role of PPARD in regulating lipid metabolism in ruminant mammary gland remains unknown. In the present study, we assessed the mRNA abundance of PPARD at 3 stages of lactation in goat mammary tissue. Results revealed that PPARD had lower expression at peak lactation than in the nonlactating period. Luciferase assays revealed that GW0742 (GW), a specific PPARD ligand, enhanced the activity of the PPARD response element in goat mammary epithelial cells. Activation of $P P A R D$ by GW selectively upregulated the expression of genes related to fatty acid activation (ACSL1), lipid droplet formation (PLIN2), and transport $(F A B P 4)$, and had no effect on genes involved in de novo fatty acid synthesis $(A C A C A$ and $F A S N)$, desaturation $(S C D)$, hydrolysis and oxidation (PNPLA2 and CPT1A), transport and uptake (FABP3 and CD36), or triacylglycerol synthesis (DGAT1 and AGPAT6) in goat mammary epithelial cells. In contrast, knockdown of PPARD using small interfering RNA dramatically decreased the expression of genes related to fatty acid activation (ACSL1) and lipid formation (PLIN2) and increased the expression of genes related to fatty acid transport (FABP3) and triacylglycerol synthesis (AGPAT6 and DGAT1). The expression of genes related to fatty acid synthesis $(F A S N)$, hydrolysis (PNPLA2), and fatty acid oxidation (CPT1A) was downregulated significantly only after knockdown of PPARD in cells incubated with GW. We observed no significant change in fatty acid profiles. However, the total cellular triacylglycerol increased after knockdown of PPARD in goat mammary epithelial cells plus GW.

\footnotetext{
Received June 21, 2016.

Accepted September 22, 2016.

${ }^{1}$ These authors contributed equally to this paper. edu

${ }^{2}$ Corresponding authors: luojun@nwsuaf.edu.cn and jloor@illinois.
}

Collectively, these results highlight an important role for PPARD in the homeostasis of ruminant mammary cells by facilitating fatty acid activation and lipid droplet formation and secretion.

Key words: goat, peroxisome proliferator-activated receptor (PPAR), milk fat, gene expression

\section{INTRODUCTION}

Peroxisome proliferator-activated receptors (PPAR) are ligand-activated transcription factors that belong to the nuclear receptor superfamily and include 3 closely related members: PPAR alpha (PPARA), gamma (PPARG), and delta (PPARD). Each member displays a tissue-selective expression pattern in ruminants, with PPARG and PPARA predominantly expressed in adipose tissue and liver, respectively, and PPARD broadly expressed in various tissues, including adipose, rumen, liver, and lung (Bionaz et al., 2013). In nonruminants, the role of PPARG in determining adipocyte differentiation and promoting lipid storage has been well established (Kawai and Rosen, 2010). Recent studies have also underscored a key role for PPARG in controlling lipid metabolism in goat mammary gland (Shi et al., 2013a; Kang et al., 2015). Similarly, a large body of evidence also supports the function of PPARA as a controller of the catabolism of fatty acids (Roberts et al., 2011; Pol et al., 2015). However, compared with PPARG and PPARA, knowledge about PPARD in the context of ruminant mammary lipid metabolism remains unknown.

In rodents, the primary role of PPARD is the control of catabolism of fatty acids in muscle (Barak et al., 2002). The expression of PPARD in skeletal muscle is increased upon fasting and upon exercise (Giordano Attianese and Desvergne, 2015), suggesting a role for PPARD in the adaptive response of skeletal muscle to increased demand for catabolism of fatty acids. Conversely, when PPARD is deleted in skeletal muscle, the muscle fibers exhibit lower oxidative activity, and body fat mass increases (Schuler et al., 2006). 
A biologic role for PPARD in the catabolism of fatty acids in white and brown adipose tissue is supported by evidence from PPARD-transgenic mice. In line with an increase in catabolism of fatty acids, transgenic mice expressing PPARD are resistant to lipid accumulation induced by high-fat diets and exhibit diminished lipidemia (Wang et al., 2003). However, the fact that mice carrying an adipose-specific deletion of $P P A R D$ did not have alterations in fat mass content was indicative of an indirect effect of PPARD in adipose tissue (Lee et al., 2006). Interestingly, the exploration of PPARDmediated response in the liver suggested that PPARD also promotes liver lipogenesis (Liu et al., 2011).

Unlike in rodents, the function of PPARD in ruminants remains largely unknown. A similar expression of PPARD has been observed in various cow tissues, and PPARD was more abundant than PPARG in cow mammary gland (Bionaz et al., 2013). Contrasting roles for PPARG and PPARD in primary bovine mammary cells has been reported, where several PPARG ligands reduced the expression of PPARD (Lutzow et al., 2008). In contrast, the fact that activation of PPARD increased the activity of glyceraldehyde-3-phosphate dehydrogenase indicated a potential role for PPARD in adipogenesis in sheep (Soret et al., 1999). The opposite responses for catabolism of fatty acids and lipogenesis in these published experiments indicate that PPARD may play different roles in different tissues. Thus, it was hypothesized that activation or knockdown of PPARD would alter the mRNA expression of genes related to lipid metabolism.

To our knowledge, few available data directly support a role for PPARD in lipid metabolism in ruminants. To investigate the effect of PPARD on lipid metabolism in ruminant mammary gland in the present study, PPARD was activated by use of a chemical ligand or was knockdown via small interfering RNA (siRNA) in goat mammary epithelial cells (GMEC).

\section{MATERIALS AND METHODS}

\section{mRNA Abundance of PPARD}

The transcriptome data set from 3 lactating goat mammary tissue samples were deposited at the National Center for Biotechnology Information (GEO Series GSE87089). Details of these samples have been described previously (Shi et al., 2015). Briefly, we used 3- to 4-yr-old Xinong Saanen dairy goats from the experimental farm of Northwest Agricultural University, Shaanxi, China. Mammary tissue at peak lactation (3 goats, $100 \mathrm{~d}$ postpartum), late lactation (cessation of milking, 3 goats, $310 \mathrm{~d}$ postpartum), and non-lactation (3 goats, nonlactating and nonpregnant) was collected by a veterinarian after slaughter. All goats were managed in a similar fashion, and were fed a mixed diet consisting of corn, soybean meal, bran, rapeseed meal, and a mineral-vitamin mixture. We used Bowtie 0.12.8 (http://bowtie-bio.sourceforge.net/index.shtml) to align reads to the goat genome (Dong et al., 2013). We used DESeq (a software for differential gene expression analysis based on negative binomial distribution of data; http://www-huber.embl.de/users/anders/DESeq) to analyze the abundance of gene expression. The single-end mapping method was used for read analysis; one read was compared with multiple genes. Reads per kilobase of exon model per million mapped reads (RPKM) were used for measuring gene expression of PPARD.

\section{Cell Culture and Treatments}

The GMEC were isolated from Xinong Saanen goats at peak lactation, as described previously (Wang et al., 2010; Shi et al., 2014). Details of cell culture were described recently (Lin et al., 2013; Shi et al., 2013b). To promote lactogenesis, GMEC were cultured in a lactogenic medium for $24 \mathrm{~h}$ before initial experiments. Cells were cultured in a 6 -well culture plate and subcultured to $90 \%$ confluence; they were then treated with PPARD-specific ligand GW0742 (GW, Sigma-Aldrich, St. Louis, MO) at $1 \mu M$ in lactogenesis medium. Then the cells were collected at 0,12 , and $24 \mathrm{~h}$ for total RNA extraction or triacylglycerol (TAG) assays. The lactogenesis medium was composed of DMEM/F12 (Hyclone, Beijing, China), insulin (5 mg/L, SigmaAldrich), hydrocortisone (5 mg/L, Sigma-Aldrich), penicillin/streptomycin (10k unit/L, Harbin Pharmaceutical Group, Harbin, China), prolactin $(2 \mu \mathrm{g} / \mathrm{mL}$, Sigma-Aldrich) and BSA (1 g/L, Sigma-Aldrich).

\section{RNA Interference}

To determine PPARD mRNA interference, cells cultured in 6-well plates were transfected with 100 $\mathrm{n} M$ siRNA and $7.5 \mu \mathrm{L}$ of transfection reagent (Lipofectamine RNAiMAX; Thermo Fisher Scientific Inc., Waltham, MA) in lactogenesis medium without antibiotic. The transfection was performed according to the manufacturer's instructions (http://tools.thermofisher. $\mathrm{com} /$ content/sfs/manuals/Lipofectamine_RNAiMAX_ Reag_protocol.pdf). Transfected GMEC were cultured with $1 \mu M$ GW (Sigma-Aldrich) or control (dimethyl sulfoxide, DMSO; Sigma-Aldrich) after $24 \mathrm{~h}$ of initial culture, and then harvested at $48 \mathrm{~h}(24 \mathrm{~h}$ later $)$ for RNA extraction, TAG assays, and fatty acid extraction. The sequences of the siRNA oligonucleotides for PPARD (accession XM_004018768.3) were 5'-GCUG- 
GCCUCCAUUGUCAAUTT-3' (designed and synthesized by Jima Biotechnology Co. Ltd., Shanghai, China). Scrambled siRNA was a functional nontargeting siRNA used as a negative control (siNC). In total, we included 4 treatments ( siNC + DMSO, siPPARD + DMSO, siNC + GW, siPPARD + GW) in the RNA interference experiment.

\section{RNA Extraction and Quantitative Real-Time PCR}

The procedures for total RNA extraction, purification, and quantitative PCR have been recently described (Lin et al., 2013). Total RNA from GMEC was extracted using the RNAprep Pure Kit (Tiangen Biotech Co. Ltd., Beijing, China) according to the manufacturer's protocol (http://www.tiangen.com/asset/imsupload/ up0203129001467351047.pdf). During RNA extraction, genomic DNA contamination was removed using DNase provided with the kit. We synthesized cDNA from $0.5 \mu \mathrm{g}$ RNA using the PrimeScript RT kit (Takara Bio Inc., Otsu, Japan) according to the manufacturer's instructions. We performed quantitative real-time PCR (qPCR) according to the manufacturer's instructions using SYBR Green (SYBR Premix Ex Taq II, Perfect Real Time; Takara Bio Inc.; http://www.clontech.com/ CN/Products/Real-Time_qPCR_and_Reverse_Transcription/Real-Time_qPCR_Probe_Detection/Premix_Ex_Taq?sitex=10022:22372:US). A melting curve was included in each $\mathrm{qPCR}$ reaction, and a positive control was included in each plate.

Genes studied included lipoprotein lipase $(L P L)$, CD36 molecule (thrombospondin receptor, CD36), carnitine palmitoyltransferase 1A (CPT1A), acyl-coA synthetase long-chain family member 1 (ACSL1), fatty acid binding protein 3 (FABP3), FABP4, fatty acid synthase $(F A S N)$, acetyl-Coenzyme A carboxylase $\alpha$ $(A C A C A)$, stearoyl-CoA desaturase 1 (SCD1), 1-acylglycerol-3-phosphate O-acyltransferase 6 (AGPAT6), diacylglycerolacyltransferase1 (DGAT1), patatin-like phospholipase domain containing 2 (PNPLA2), perilipin2 (PLIN2), and PPARD. All qPCR reactions were performed in a Bio-Rad CFX96 (Bio-Rad Laboratories Inc., Hercules, CA) sequence detector, and data were normalized to ubiquitously expressed transcript ( UXT), mitochondrial ribosomal protein L39 (MRPL39), and ribosomal protein $\mathrm{S} 9$ (RPS9). The sequences of the primers for the genes are described in Supplementary Table S1 (https://doi.org/10.3168/jds.2016-11647).

\section{Fatty Acid Analysis}

Collected GMEC were washed 3 times with PBS and then scraped off the culture dish using a $2-\mathrm{mL}$ aliquot of $2.5 \%$ ( $\mathrm{vol} / \mathrm{vol}$ ) vitriol:methanol. Then, we performed total lipid extraction and methylation according to Shi et al. (2013a). Methylated lipid samples were analyzed using a gas chromatography-mass spectrometer (Agilent Technologies, Santa Clara, CA) installed with an HP-5 column $(35 \mathrm{~m} \times 0.25 \mathrm{~mm}$ i.d. $\times 0.25 \mu \mathrm{m}$ film thickness) following a published procedure (Yao et al., 2016). The relative proportion of each fatty acid was determined as the ratio of the fatty acid peak to the total peaks in each run. The data for each fatty acid were analyzed as a proportion of the total fatty acids.

\section{Cellular TAG Assays}

Total cellular TAG was extracted according to the protocol of the GPO-Trinder triglyceride assay kit (Applygen Technologies, Beijing, China; http://www. applygen.com/a/meixueyushenghuaceding/276.html).

The concentration of TAG was determined according to the manufacturer's instructions on a microtiter plate reader (BioTek, Winooski, VT). We calculated the concentrations using the equation obtained from linear regression of the standard curve. The quantification of total cellular TAG was normalized to the cellular protein concentration. We determined the protein concentration for each well using a bicinchoninic protein assay (Pierce; Thermo Fisher Scientific, Waltham, MA) according to the manufacturer's instructions.

\section{Luciferase Activity Analysis}

The GMEC at $80 \%$ confluence in 48-well plates were transfected with $0.2 \mu \mathrm{g}$ of PPARD response element $($ PPRE $) \times 3$-Luc reporter plasmid along with a Renilla vector (pRL-TK) as a control at a ratio of 25:1 using the Lipofectamine 2000 (Thermo Fisher Scientific Inc.). After a 24-h recovery period in lactogenesis medium, one group of cells was treated with GW at final concentrations of $0,0.1,1,5$, and $10 \mu M$ and harvested after $48 \mathrm{~h}$. The other group was treated with $\mathrm{GW}$ at $1 \mu M$ (final concentration), and cells were harvested after 0,12 , and $24 \mathrm{~h}$, respectively. Lysates were made using reporter lysis buffer (Dual-Luciferase Reporter Assay System, Promega, Madison, WI) according to the manufacturer's instructions. Luciferase activity in the cell extract was determined using luciferase assay buffer and luciferase assay substrate according to the manufacturer's protocol.

\section{Statistical Analysis}

Treatments were replicated at least 3 times in culture wells, and results were expressed as mean \pm standard error of the mean. Data for qPCR were analyzed us- 
ing the $2^{-\Delta \Delta \mathrm{Ct}}$ method. Data for $\mathrm{qPCR}$ in cells treated only with GW were analyzed relative to the control $(0 \mathrm{~h})$ using one-way ANOVA. All data for qPCR were normalized to 3 internal control genes (UXT, MRPL39, and RPS9). The significance of relative luciferase activity across different concentrations and times was determined by one-way ANOVA. The significance of RNA expression after PPARD knockdown was determined by one-way ANOVA. Significance was declared at $P$ $<0.05$.

\section{RESULTS}

\section{PPARD Expression in Dairy Goat Mammary Gland Tissue}

To assess the expression of PPARD in goat mammary gland tissue, we evaluated RNA sequencing data from 3 stages of lactation. As shown in Figure 1, the nonlactating mammary gland had the highest RPKM value for PPARD, followed by the late-lactation samples. Compared with the nonlactating period, we detected a lower expression of PPARD at peak lactation $(P<$ $0.05)$.

\section{A PPARD Ligand Enhanced Activity of the PPAR Response Element in GMEC}

As shown in Figure 2, treatments with GW caused a dramatic activation of PPARD in GMEC. The luciferase levels between each treatment group and the control group (treatment with $0 \mu M \mathrm{GW}$ ) increased significantly $(P<0.05)$. Activation of PPARD by GW reached a peak at the $5 \mu M$ dose ( $\sim 4$-fold; Figure $2 \mathrm{~A})$.

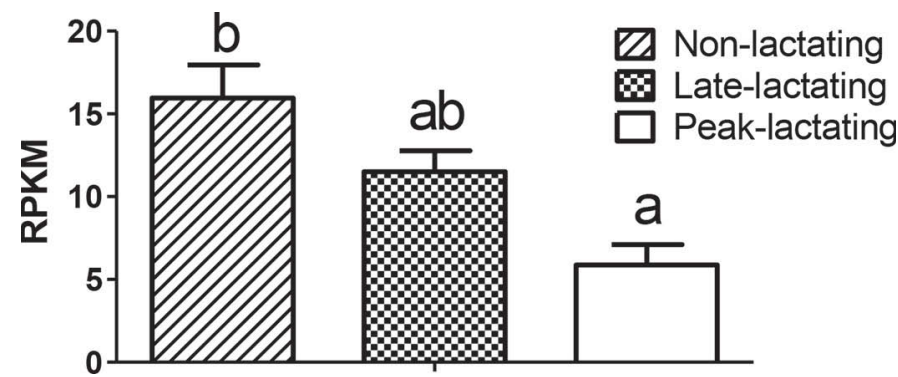

Figure 1. The mRNA abundance of peroxisome proliferator-activated receptor delta $(P P A R D)$ at 3 stages of lactation in goat mammary tissues. The transcriptome data set is from mammary tissue harvested at peak lactation (3 goats, $100 \mathrm{~d}$ postpartum), in late lactation (cessation of milking, 3 goats, $310 \mathrm{~d}$ postpartum), and non-lactation (3 goats, nonlactating and nonpregnant) by a veterinarian after slaughter. The DESeq software http://www-huber.embl.de/users/anders/DESeq was used to analyze abundance of gene expression. Reads per kilobase of exon model per million mapped reads (RPKM) was used to assess gene expression. Values are means \pm SEM for 3 individual goats. The different letters $(\mathrm{a}, \mathrm{b})$ denote significant $(P<0.05)$ differences.

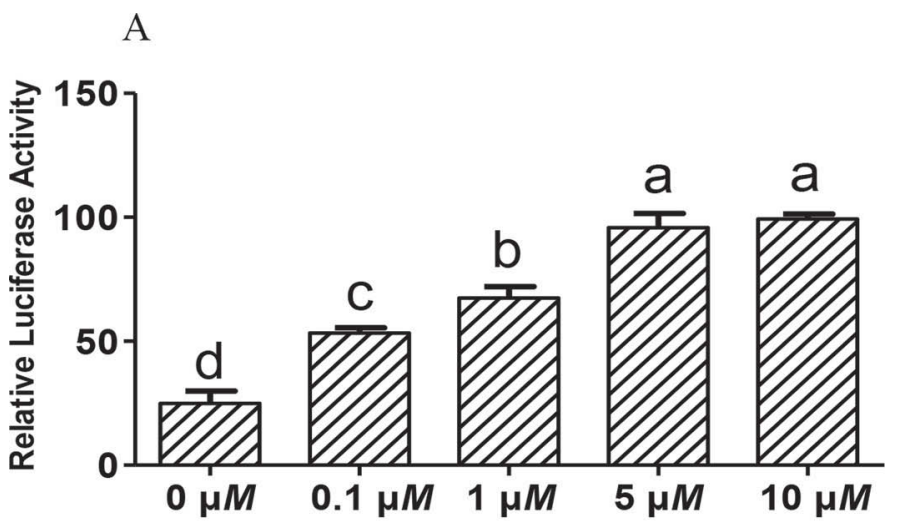

B

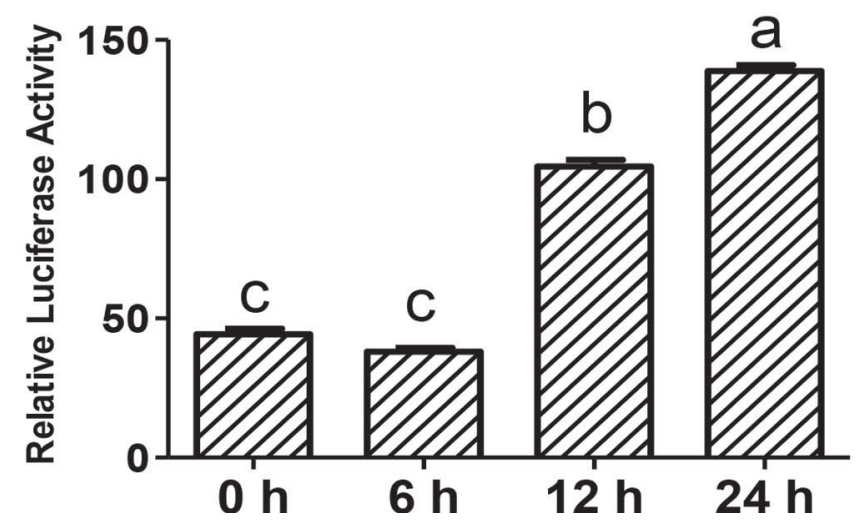

Figure 2. The peroxisome proliferator-activated receptor delta (PPARD)-specific ligand GW0742 (GW) ligand activated the PPARD response element (PPRE) effectively in goat mammary epithelial cells. Cells were transfected with pGL3-PPRE $\times 3$ and pRL-TK vectors. (A) After transfection, cells were treated with GW at $0,0.1,1,5$, and $10 \mu M$ for $24 \mathrm{~h}$, respectively. (B) Transfected cells at a $1-\mu M$ dose were collected at $0,6,12$, and $24 \mathrm{~h}$ for luciferase assays. Luciferase and Renilla luciferase assays were performed in triplicate. Relative luciferase activity was expressed relative to control $(0 \mu M)$ or $0 \mathrm{~h}$ and normalized with Renilla luciferase activity, respectively. Values are mean \pm SEM from 3 independent experiments. The different letters (a to d) denote significant $(P<0.05)$ differences in relative luciferase activity.

The relative luciferase activity increased $\sim 3$-fold at 24 $\mathrm{h}$ in GMEC treated with $1 \mu M \mathrm{GW}$.

\section{Activation of PPARD by GW Affects Expression of Genes Related to Fatty Acid Metabolism in GMEC}

Compared with the control group (cells treated with GW at $0 \mathrm{~h}$ ), GW had no significant effect on the mRNA expression of PPARD (Figure 3A). Among the genes related to de novo synthesis and desaturation, activation of PPARD by GW had no effect on $A C A C A$, $F A S N$, or $S C D 1$ (Figure 3B). We observed no effect on PNPLA2, CPT1A, DGAT1, or AGPAT6 after activation of PPARD by GW (Figures $3 \mathrm{C}$ and $\mathrm{E}$ ). In contrast, 
activation of PPARD by GW led to significant upregulation of ACSL1 at 12 and $24 \mathrm{~h}$ (Figure 3C). Among the genes related to fatty acid transport and uptake, $F A B P 4$ was upregulated $(P<0.05)$ by treatment with GW (Figure 3D). We observed a dramatic upregulation of PLIN2 in cells incubated with GW at 12 and $24 \mathrm{~h}$ $(\sim 10$ - and $\sim 14$-fold, respectively, $P<0.01)$.

\section{Knockdown of PPARD Affects the Expression of Genes Related to Fatty Acid Metabolism in GMEC}

As shown in Figure 4A, treatment of GMEC with siRNA reduced PPARD mRNA abundance by $85 \%$. Compared with the control group (siNC + DMSO), knockdown of PPARD (siPPARD + DMSO) did not significantly alter the mRNA abundance of $A C A C A$, FASN, SCD1, PNPLA2, CPT1A, CD36, or FABP4; however, it did increase FABP3 $(P=0.023)$, AGPAT6 $(P=0.04)$, and DGAT1 $(P=0.023$; Figures $4 \mathrm{D}$ and E). Knockdown of PPARD in cells cultured with GW downregulated the mRNA abundance of FASN $(P=$ 0.035), PNPLA2 $(P=0.022)$, and CPT1A $(P=0.047$; Figures $4 \mathrm{~B}$ and $\mathrm{C})$. It is noteworthy that the pattern of expression of PLIN2 and ACSL1 was strongly consistent with the activation and knockdown of PPARD (Figures $4 \mathrm{C}$ and E). Treatment of GMEC with PPARD siRNA significantly downregulated both ACSL1 and PLIN2, regardless of GW.

\section{PPARD Alters Cellular TAG but Fails to Change Fatty Acid Composition in GMEC}

Compared with DMSO, incubation with GW for $24 \mathrm{~h}$ did not alter the cellular TAG profile in GMEC (Figure 5). We also observed no significant change in TAG in cells treated with PPARD siRNA (siPPARD + DMSO) compared with the control group (siNC + DMSO). However, knockdown of PPARD in GMEC incubated with GW increased the total cellular TAG $(P<0.05$; Figure 5). The results of fatty acid analysis illustrated that knockdown or activation of PPARD did not affect the ratio of $\mathrm{C} 14, \mathrm{C} 16: 0, \mathrm{C} 16: 1, \mathrm{C} 18: 0, \mathrm{C} 18: 1 \mathrm{n}-7$, or C18:1n-9 in GMEC (Figure 6).

\section{DISCUSSION}

In ruminant mammary gland, recent data have strongly underscored the role of PPARG in lipogenesis and TAG secretion via control of gene expression (Shi et al., 2013a; Kang et al., 2015). The fact that the relative mRNA abundance of $P P A R D$ is higher than that of PPARG in bovine mammary tissue (Bionaz et al., 2013) led us to hypothesize that PPARD also plays an important role in mammary gland lipid metabolism.
The present study is novel in that we studied the selective regulation of gene expression and cellular TAG content by PPARD in GMEC using a combination of activation with GW and knockdown via siRNA. The findings from the present study underscore an important role for PPARD in regulating the transcription of PLIN2 and ACSL1, which are related to TAG synthesis and lipid droplet formation, 2 key processes required for milk fat secretion.

The markedly lower expression of PPARD at peak lactation compared with the dry period indicated an opposite role for PPARD compared with PPARG during lactation. Together with the fact that PPARG expression in lactating goat mammary tissue is higher than in the dry period (Shi et al., 2013a), the present data agree with the observation that several PPARG ligands decreased the expression of PPARD in primary bovine mammary cells (Lutzow et al., 2008). The present data also support a previous hypothesis that PPARD may perform a role opposite to PPARG (Bionaz et al., 2013). Clearly, additional experiments are needed to better define the mechanisms of PPARD biology in the ruminant mammary gland.

Once activated by a ligand, PPARD forms a heterodimer with retinoid X receptor and binds to PPRE in the promoter region of its target genes (Tan et al., 2005). The marked increase in luciferase levels upon incubation with GW indicated that PPARD activation was ligand-dependent. The higher level of luciferase induced by GW in this study compared with that induced by rosiglitazone (Shi et al., 2013b) indicates that PPARD is more readily activated than PPARG in GMEC. Such a response may be partly due to the higher expression of PPARD demonstrated in bovine mammary tissue (Bionaz et al., 2013). The higher activation of PPARD in mammary tissue strongly underscores its importance in ruminant mammary tissue.

The proteins encoded by $A C A C A, F A S N$, and $S C D$ play an important role in de novo fatty acid synthesis in the mammary gland. The lack of change in expression of $A C A C A, F A S N$, and $S C D 1$ with GW agree with data from PPARD-transgenic mice (Wang et al., 2003). The downregulation of FASN after knockdown of PPARD in GMEC incubated with GW indicated that PPARD elicits some control over fatty acid synthesis, at least in GMEC. From a mechanistic standpoint, the alteration of FASN upon PPARD activation may be functionally related to the unique characteristics of ruminant mammary cells. This hypothesis is supported by the marked increase in rates of fatty acid synthesis in lactating ruminant mammary gland, which is required for milk fat synthesis and secretion (Mellenberger et al., 1973).

The lack of change in $\mathrm{C} 16: 1$ and $\mathrm{C} 18: 1$ percentage in this study agrees with the lack of change in $S C D 1$ with 
A


B

C
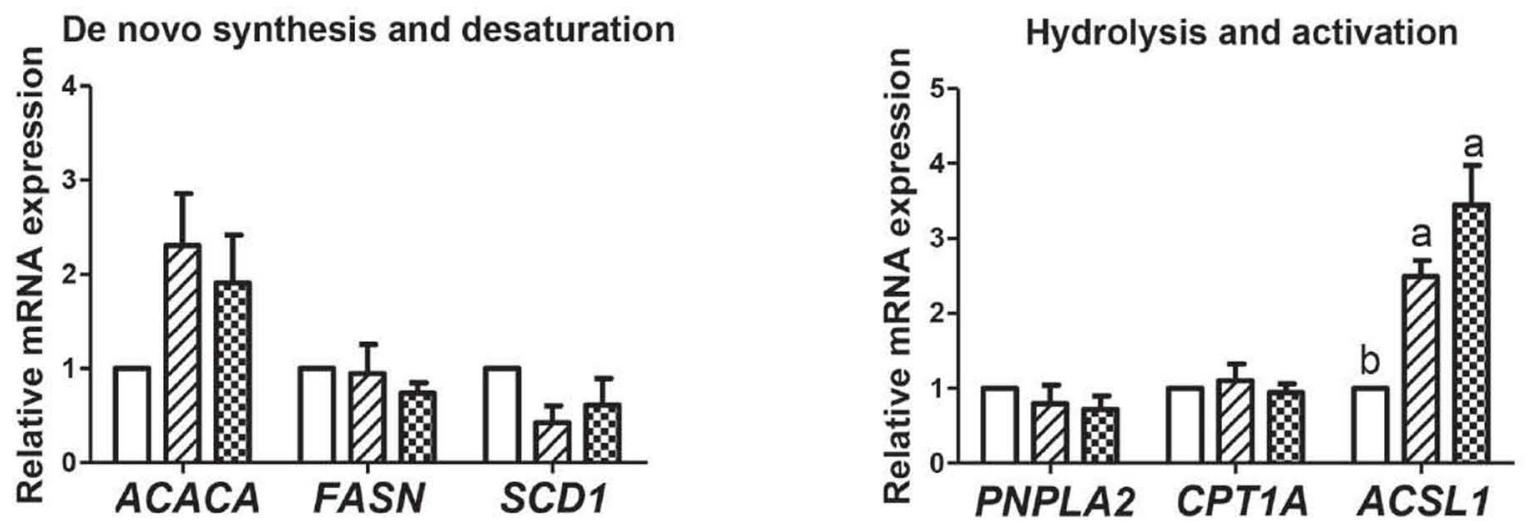

D

$\mathrm{E}$

Fatty acid transport and uptake

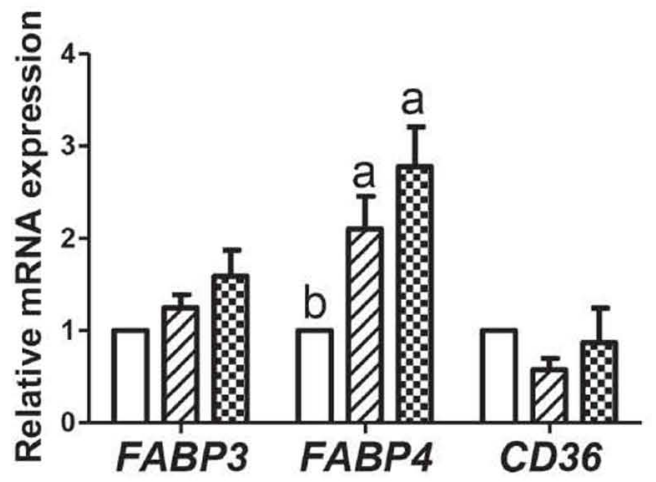

Triacylglycerol synthesis and formation

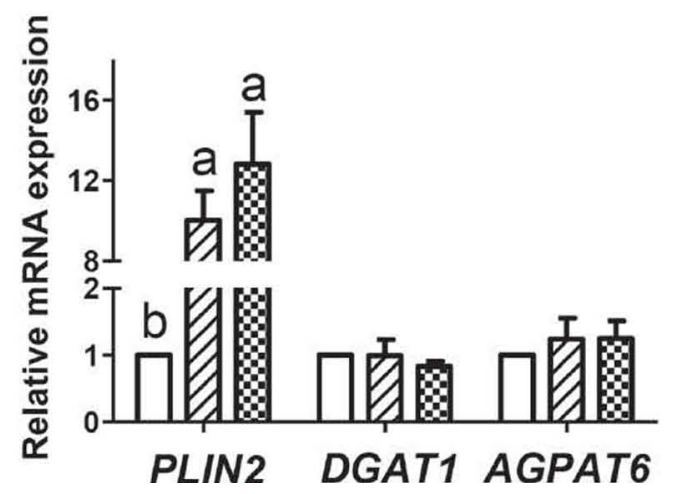

Figure 3. Activation of peroxisome proliferator-activated receptor delta (PPARD) by GW0742 (GW) alters the expression of genes coding for proteins related to lipid metabolism in goat mammary epithelial cells. Cells were incubated with GW at $1 \mu M$ and harvested after 0 , 12 , and $24 \mathrm{~h}$ to assess gene expression. Panels show the mRNA expression of (A) PPARD; (B) genes related to de novo synthesis (ACACA and $F A S N$ ) and desaturation (SCD1); (C) genes related to triacylglycerol hydrolysis (PNPLA2 and CPT1A) and fatty acid activation (ACSL1); (D) genes related to fatty acid transport (FABP3 and FABP4) and uptake (CD36); and (E) genes related to triacylglycerol synthesis (DGAT1 and $A G P A T 6)$ and lipid droplet formation (PLIN2). Values are means \pm SEM from 3 individual cultures. The different letters denote significant $(P$ $<0.05)$ differences. 


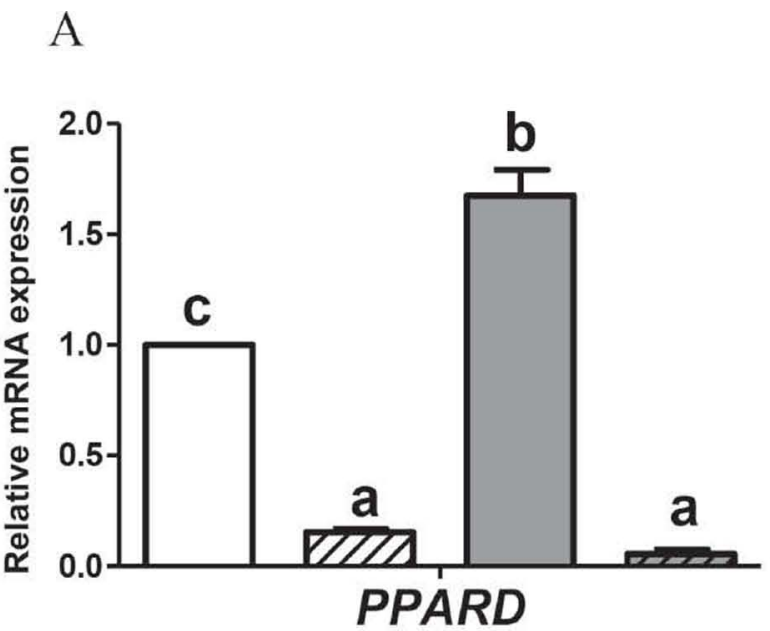

$\square$ siNC + DMSO $\square$ SiPPARD + DMSO $\square$ siNC + GW $\square$ siPPARD + GW
B

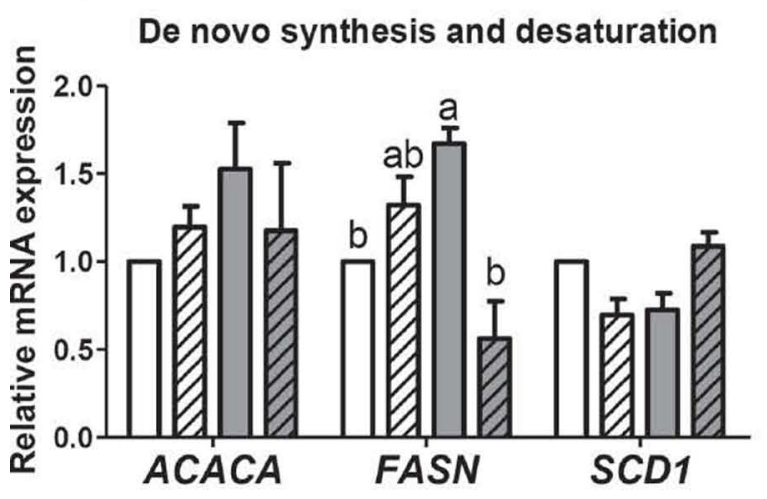

D

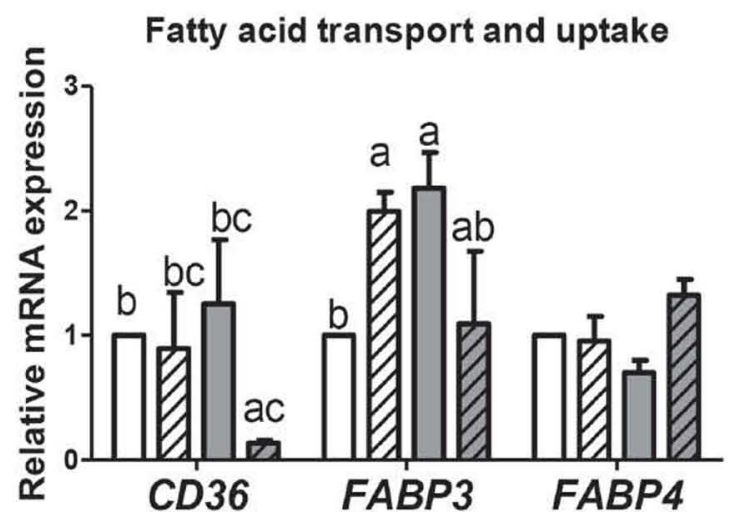

C

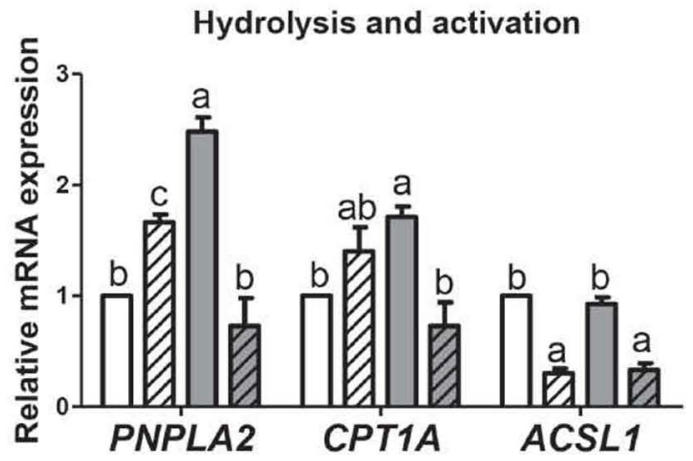

E

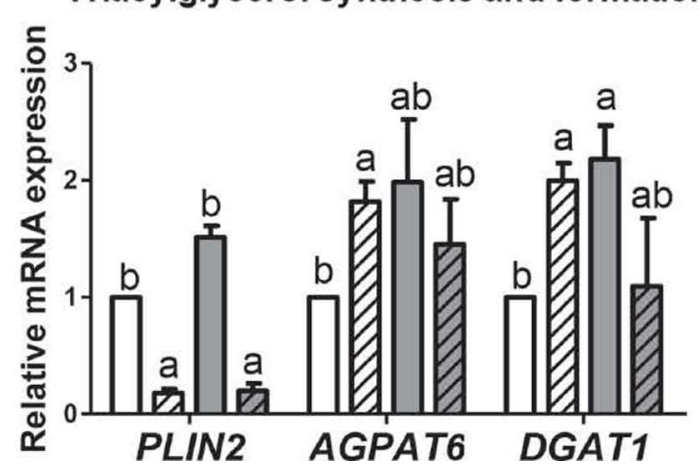

Figure 4. Changes in gene expression in goat mammary epithelial cells in response to knockdown of peroxisome proliferator-activated receptor delta $[P P A R D$; siPPARD + DMSO (dimethyl sulfoxide)], incubation with PPARD-specific ligand GW0742 (GW; siNC + GW), or knockdown of PPARD plus GW (siPPARD + GW). Panels show the mRNA expression of (A) PPARD; (B) genes related to de novo synthesis ( $A C A C A$ and $F A S N$ ) and desaturation (SCD1); (C) genes related to triacylglycerol hydrolysis (PNPLA2 and $C P T 1 A)$ and fatty acid activation (ACSL1); (D) genes related to fatty acid transport (FABP3 and FABP4) and uptake (CD36); and (E) genes related to triacylglycerol synthesis (DGAT1 and AGPAT6) and lipid droplet formation (PLIN2). Values are means \pm SEM from 3 individual cultures. Two comparisons (siPPARD + DMSO vs. siNC + DMSO and siPPARD + GW vs. siNC + GW $)$ were performed. The different letters $($ a to c $)$ denote significant $(P<0.05)$ differences. 


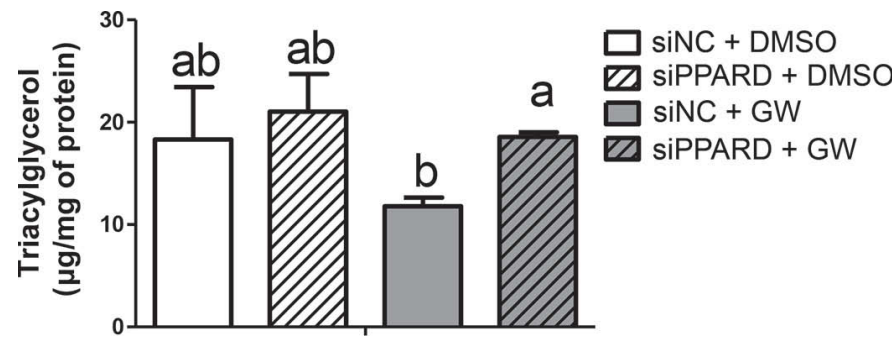

Figure 5. Changes in total cellular triacylglycerol in goat mammary epithelial cells in response to knockdown of peroxisome proliferatoractivated receptor delta $[P P A R D$; siPPARD + DMSO (dimethyl sulfoxide)], incubation with PPARD-specific ligand GW0742 (GW; siNC $+\mathrm{GW}$ ), incubation control and GW (siNC + DMSO), or knockdown of PPARD plus GW (siPPARD + GW). Data are means \pm SEM from 3 individual cultures. The different letters $(\mathrm{a}, \mathrm{b})$ denote significant $(P$ $<0.05)$ differences.

both GW treatment and knockdown of PPARD. The fact that $S C D 1$ is a verified target of PPARG in GMEC (Shi et al., 2013a) indicates that target gene regulation by PPARD is mediated via a different PPRE (Tan et al., 2005).

Previous work with nonruminants has clearly identified PPARD as an activator of genes required for oxidation in brown adipocyte tissue. The decrease in expression of PNPLA2 and CTP1A after PPARD knockdown and GW supplementation indicates that PPARD is not required for the basal transcription of genes associated with the catabolism of fatty acids in ruminant mammary gland. A similar transcriptional regulatory mechanism in bovine mammary cells has also been reported for other transcription factors, such as liver X receptor $\alpha$ (Oppi-Williams et al., 2013).

The dynamic activation of PPARD seems to participate in balancing mammary cell homeostasis to avoid excessive lipogenesis, or to control it. In the physiological context of milk fat synthesis in lactating mammary gland, this mechanism is not far-fetched, given that the lactating mammary cell must synthesize TAG and export it to ensure that intracellular TAG does not accumulate. Several knockout studies in mouse have demonstrated that excessive intracellular TAG accumulation could be toxic to mammary epithelial cells and elicit a negative effect on lactation (Ogg et al., 2004; Beigneux et al., 2006).

The putative function of PPARD to prevent excessive lipogenesis also is supported by the response of PLIN2 in cultures incubated with GW or with the PPARD knockdown using siRNA. The PLIN2 protein is essential for the formation and secretion of lipid droplets during lactation (Chong et al., 2011; Russell et al., 2011). The dramatic changes in PLIN2 in response to GW (present study) or the overexpression of PPARD in adipose tissue (Wang et al., 2003) agree with the notion that PLIN2 is a target of PPARD. Our recent data demonstrated that PPARG directly regulates PLIN2
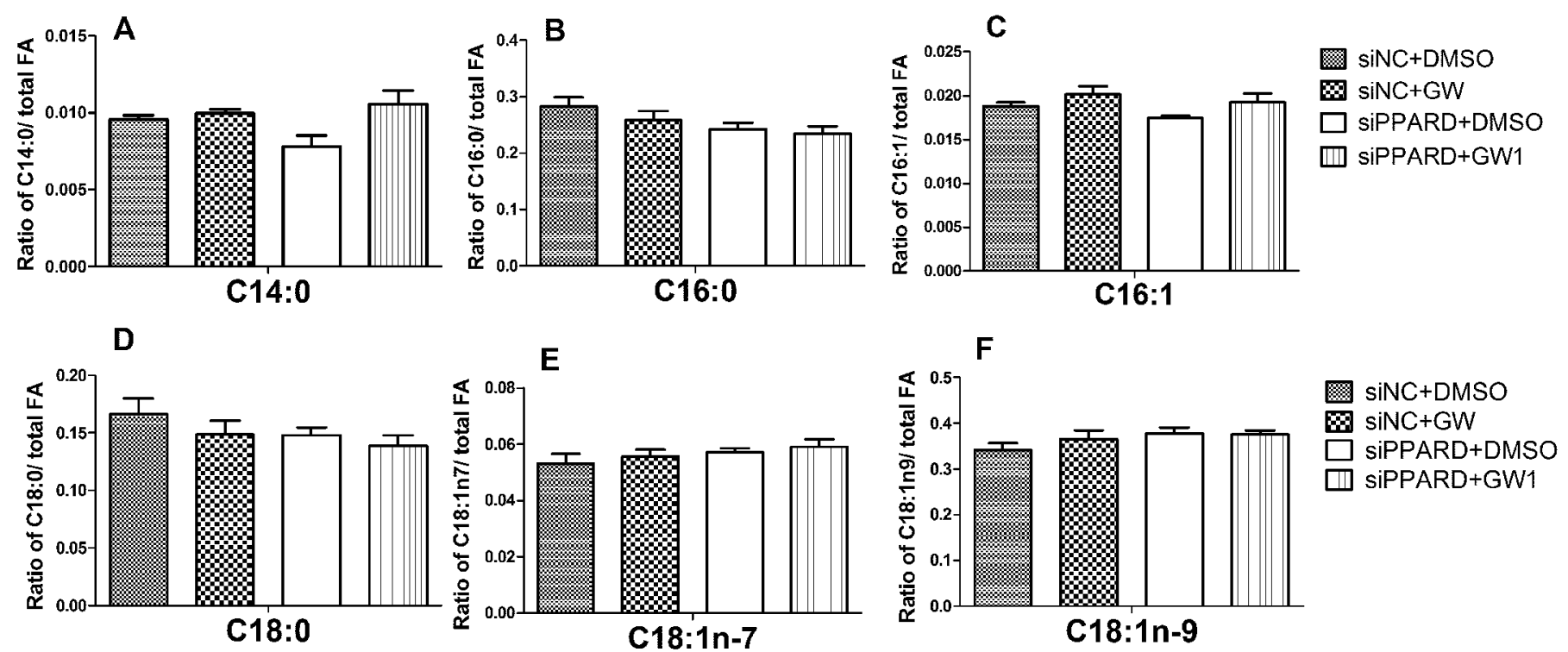

Figure 6. Knockdown of peroxisome proliferator-activated receptor delta (PPARD) did not alter the fatty acid (FA) composition of goat mammary epithelial cells. Panels A, B, C, D, E, and F depict the percentage of C14:0, C16:0, C16:1, C18:0, C18:1n-7, and C18:1n-9, respectively, in goat mammary epithelial cells in response to knockdown of PPARD [siPPARD + DMSO (dimethyl sulfoxide)], incubation with PPARD-specific ligand GW0742 alone (siNC + GW), incubation with control and DMSO (siNC+DMSO), or knockdown of PPARD plus GW $($ siPPARD + GW $)$. Data are means \pm SEM for 3 individual experiments. No significant change were observed among the comparisons using one-way ANOVA. 
by binding to the PPRE site on the promoter (Kang et al., 2015). Whether the 2 transcription factors share the same PPRE is still unknown. However, the lack of significant change in cellular TAG in response to GW or knockdown of PPARD indicates a potential balance or concerted action between PPARG and PPARD on PLIN2 to regulate the synthesis of lipid droplets in mammary cells.

Although PLIN2 certainly is not the only protein that could alter lipid droplet formation, it does determine lipid droplet formation in mammary cells (Shi, et al., 2015). However, other proteins such as tail-interacting 47-kDa protein and cell death-inducing DFF45-like effector family also play a role. The observed regulation of PLIN2 by PPARD in the present study also helps explain the fact that $P L I N 2$ expression remained high even after knockdown of PPARG in GMEC (Shi et al., 2013b). Collectively, the findings in the present study underscore a central role for PPARD in the regulation of milk lipid droplet secretion.

The induction of $F A B P 4$ by $\mathrm{GW}$ in the present study is consistent with the fact that there are 2 PPREs in the promoter region of bovine FABP4 (Shin et al., 2009). The CD36 protein is an essential plasma membrane transporter that allows for fatty acid uptake by mammary cells (Bionaz and Loor, 2008). Despite the fact that there are PPRE sites on its promoter, the lack of change in $C D 36$ expression with supplementation of GW and knockdown of PPARD indicates that it is not a target gene of PPARD in ruminant mammary cells (Zhou et al., 2008).

Long-chain fatty acids are esterified with $\mathrm{CoA}$ in the inner face of the plasma membrane before participating in metabolic pathways. The ACSL1 gene is predominant among ACSL isoforms in bovine mammary tissue (Bionaz and Loor, 2008) and is considered a key enzyme in activating fatty acids destined for TAG synthesis and $\beta$-oxidation (Li et al., 2009). Recent data have also demonstrated that ACSL1 could enhance fatty acid synthesis in bovine mammary cells (Lian et al., 2016). In the present study, the alteration of ACSL1 mRNA abundance response to activation and knockdown of PPARG strongly suggest that ACSL1 is a target of PPARD in ruminant mammary cells. The fact that activation or knockdown of PPARD did not change the total cellular TAG is consistent with observations in mouse liver, where the content of TAG was unchanged in liver of $A \operatorname{cs} 11^{-/-}$mice after feeding either low- or high-fat diets (Li et al., 2009). The higher cellular TAG after knockdown of PPARD plus GW compared with control (siNC-GW) might have been due to a decrease in TAG hydrolysis, which was partly confirmed by the downregulation of PNPLA2 and CPT1A upon PPARD knockdown and GW supplementation. In contrast, the upregulation of AGPAT6 and DGAT1 upon the knockdown of PPARD is consistent with the fact that knockout of ACSL1 diminished the incorporation of $\left[{ }^{14} \mathrm{C}\right]$ oleate into TAG ( $\mathrm{Li}$ et al., 2009). Thus, the data indicate that PPARD suppresses cellular TAG biosynthesis.

Collectively, our data highlight a role for PPARD in facilitating the formation and secretion of lipid droplets in goat mammary cells. This role seems to extend to the suppression of biosynthesis of cellular TAG. The induction of PNPLA2 and CPT1A supports a role for PPARD in the catabolism of fatty acids. The mechanism leading to this function likely involves the regulation of ACSL1 and PLIN2.

\section{CONCLUSIONS}

In the present study, the combination of ligand activation and gene knockdown using siRNA underscore a novel role for PPARD in homeostasis in ruminant mammary cells by facilitating the catabolism of fatty acids, as well as lipid droplet formation and secretion. More controlled molecular experiments will have to be performed to address questions about the precise signaling pathway of PPARD. Together, results from the present study indicate a crucial role for PPARD in the regulation of milk fat synthesis.

\section{ACKNOWLEDGMENTS}

This research was jointly supported by a Zhejiang Sci-Tech University Grant (Hangzhou, China) and the Transgenic New Species Breeding Program of China (Beijing, China; 2014ZX08009-051B).

\section{REFERENCES}

Barak, Y., D. Liao, W. He, E. S. Ong, M. C. Nelson, J. M. Olefsky, R. Boland, and R. M. Evans. 2002. Effects of peroxisome proliferatoractivated receptor $\delta$ on placentation, adiposity, and colorectal cancer. Proc. Natl. Acad. Sci. USA 99:303-308.

Beigneux, A. P., L. Vergnes, X. Qiao, S. Quatela, R. Davis, S. M. Watkins, R. A. Coleman, R. L. Walzem, M. Philips, K. Reue, and S. G. Young. 2006. Agpat6-A novel lipid biosynthetic gene required for triacylglycerol production in mammary epithelium. J. Lipid Res. 47:734-744.

Bionaz, M., S. Chen, M. J. Khan, and J. J. Loor. 2013. Functional role of PPARs in ruminants: Potential targets for fine-tuning metabolism during growth and lactation. PPAR Res. 2013:684159.

Bionaz, M., and J. Loor. 2008. Gene networks driving bovine milk fat synthesis during the lactation cycle. BMC Genomics 9:366.

Chong, B. M., P. Reigan, K. D. Mayle-Combs, D. J. Orlicky, and J. L. McManaman. 2011. Determinants of adipophilin function in milk lipid formation and secretion. Trends Endocrinol. Metab. $22: 211-217$

Dong, Y., M. Xie, Y. Jiang, N. Q. Xiao, X. Y. Du, W. G. Zhang, G. Tosser-Klopp, J. H. Wang, S. Yang, J. Liang, W. B. Chen, J. Chen, P. Zeng, Y. Hou, C. Bian, S. K. Pan, Y. X. Li, X. Liu, W. L. Wang, B. Servin, B. Sayre, B. Zhu, D. Sweeney, R. Moore, W. H. Nie, Y. Y. Shen, R. P. Zhao, G. J. Zhang, J. Q. Li, T. Faraut, J. Womack, Y. P. Zhang, J. Kijas, N. Cockett, X. Xu, S. H. Zhao, 
J. Wang, and W. Wang. 2013. Sequencing and automated wholegenome optical mapping of the genome of a domestic goat (Capra hircus). Nat. Biotechnol. 31:135-141.

Giordano Attianese, G. M. P., and B. Desvergne. 2015. Integrative and systemic approaches for evaluating PPAR $\beta / \delta$ (PPARD) function. Nucl. Recept. Signal. 13:e001.

Kang, Y., S. Hengbo, L. Jun, L. Jun, Z. Wangsheng, T. Huibin, and S. Huaiping. 2015. PPARG modulated lipid accumulation in dairy GMEC via regulation of ADRP gene. J. Cell. Biochem. 116:192201.

Kawai, M., and C. J. Rosen. 2010. PPAR gamma: A circadian transcription factor in adipogenesis and osteogenesis. Nat. Rev. Endocrinol. 6:629-636.

Lee, C. H., P. Olson, A. Hevener, I. Mehl, L. W. Chong, J. M. Olefsky, F. J. Gonzalez, J. Ham, H. Kang, J. M. Peters, and R. M. Evans. 2006. PPARdelta regulates glucose metabolism and insulin sensitivity. Proc. Natl. Acad. Sci. USA 103:3444-3449.

Li, L. O., J. M. Ellis, H. A. Paich, S. Wang, N. Gong, G. Altshuller, R. J. Thresher, T. R. Koves, S. M. Watkins, D. M. Muoio, G. W. Cline, G. I. Shulman, and R. A. Coleman. 2009. Liver-specific loss of long chain acyl-CoA synthetase-1 decreases triacylglycerol synthesis and beta-oxidation and alters phospholipid fatty acid composition. J. Biol. Chem. 284:27816-27826.

Lian, S., J. R. Guo, X. M. Nan, L. Ma, J. J. Loor, and D. P. Bu. 2016. MicroRNA Bta-miR-181a regulates the biosynthesis of bovine milk fat by targeting ACSL1. J. Dairy Sci. 99:3916-3924.

Lin, X. Z., J. Luo, L. Zhang, W. Wang, H. Shi, and J. Zhu. 2013. MiR27a suppresses triglyceride accumulation and affects gene mRNA expression associated with fat metabolism in dairy goat mammary gland epithelial cells. Gene 521:15-23.

Liu, S., B. Hatano, M. Zhao, C. C. Yen, K. Kang, S. M. Reilly, M. R Gangl, C. Gorgun, J. A. Balschi, J. M. Ntambi, and C. H. Lee. 2011. Role of peroxisome proliferator-activated receptor $\{$ delta\}/ \{beta\} in hepatic metabolic regulation. J. Biol. Chem. 286:12371247.

Lutzow, Y. S., C. Gray, and R. Tellam. 2008. 15-Deoxy- $\Delta 12,14$ prostaglandin J2 induces chemokine expression, oxidative stress and microfilament reorganization in bovine mammary epithelial cells. J. Dairy Res. 75:55-63.

Mellenberger, R. W., D. E. Bauman, and D. R. Nelson. 1973. Metabolic adaptations during lactogenesis. Fatty acid and lactose synthesis in cow mammary tissue. Biochem. J. 136:741-748.

Ogg, S. L., A. K. Weldon, L. Dobbie, A. J. H. Smith, and I. H. Mather 2004. Expression of butyrophilin (Btn1a1) in lactating mammary gland is essential for the regulated secretion of milk-lipid droplets. Proc. Natl. Acad. Sci. USA 101:10084-10089.

Oppi-Williams, C., J. K. Suagee, and B. A. Corl. 2013. Regulation of lipid synthesis by liver X receptor alpha and sterol regulatory element-binding protein 1 in mammary epithelial cells. J. Dairy Sci. 96:112-121.

Pol, C. J., M. Lieu, and K. Drosatos. 2015. PPARs: Protectors or opponents of myocardial function? PPAR Res. 2015:835985.

Roberts, L. D., A. J. Murray, D. Menassa, T. Ashmore, A. W. Nicholls, and J. L. Griffin. 2011. The contrasting roles of PPAR $\delta$ and
PPAR $\gamma$ in regulating the metabolic switch between oxidation and storage of fats in white adipose tissue. Genome Biol. 12:R75.

Russell, T. D., J. Schaack, D. J. Orlicky, C. Palmer, B. H.-J. Chang, L. Chan, and J. L. McManaman. 2011. Adipophilin regulates maturation of cytoplasmic lipid droplets and alveolae in differentiating mammary glands. J. Cell Sci. 124:3247-3253.

Schuler, M., F. Ali, C. Chambon, D. Duteil, J.-M. Bornert, A. Tardivel, B. Desvergne, W. Wahli, P. Chambon, and D. Metzger. 2006 PGC1A expression is controlled in skeletal muscles by PPARbeta, whose ablation results in fiber-type switching, obesity, and type 2 diabetes. Cell Metab. 4:407-414.

Shi, H., H. Shi, J. Luo, W. Wang, A. B. Haile, H. Xu, and J. Li. 2014 Establishment and characterization of a dairy goat mammary epithelial cell line with human telomerase (hT-MECs). Anim. Sci. J. 85:735-743.

Shi, H., J. Zhu, J. Luo, W. Cao, H. Shi, D. Yao, J. Li, Y. Sun, H. Xu, $\mathrm{K}$. Yu, and J. Loor. 2015. Genes regulating lipid and protein metabolism are highly expressed in mammary gland of lactating dairy goats. Funct. Integr. Genomics 15:309-321.

Shi, H. B., J. Luo, D. W. Yao, J. J. Zhu, H. F. Xu, H. P. Shi, and J. J. Loor. 2013a. Peroxisome proliferator-activated receptor-Y stimulates the synthesis of monounsaturated fatty acids in dairy goat mammary epithelial cells via the control of stearoyl-coenzyme A desaturase. J. Dairy Sci. 96:7844-7853.

Shi, H., J. Luo, J. J. Zhu, J. Li, Y. T. Sun, X. Z. Lin, L. P. Zhang, D. W. Yao, and H. P. Shi. 2013b. PPAR $\gamma$ regulates genes involved in triacylglycerol synthesis and secretion in mammary gland epithelial cells of dairy goats. PPAR Res. 2013:310948.

Shin, J., B. Li, M. E. Davis, Y. Suh, and K. Lee. 2009. Comparative analysis of fatty acid-binding protein 4 promoters: Conservation of peroxisome proliferator-activated receptor binding sites. J. Anim. Sci. 87:3923-3934.

Soret, B., H. Lee, E. Finley, S. Lee, and R. Vernon. 1999. Regulation of differentiation of sheep subcutaneous and abdominal preadipocytes in culture. J. Endocrinol. 161:517-524.

Tan, N. S., L. Michalik, B. Desvergne, and W. Wahli. 2005. Multiple expression control mechanisms of peroxisome proliferator-activated receptors and their target genes. J. Steroid Biochem. Mol. Biol. 93:99-105.

Wang, Y. X., C. H. Lee, S. Tiep, R. T. Yu, J. Ham, H. Kang, and R. M. Evans. 2003. Peroxisome-proliferator-activated receptor $\delta$ activates fat metabolism to prevent obesity. Cell 113:159-170.

Wang, Z., J. Luo, W. Wang, W. Zhao, and X. Lin. 2010. Characterization and culture of isolated primary dairy goat mammary gland epithelial cells. Sheng Wu Gong Cheng Xue Bao 26:1123-1127.

Yao, D. W., J. Luo, Q. Y. He, M. Wu, H. B. Shi, H. Wang, M. Wang, H. F. Xu, and J. J. Loor. 2016. Thyroid hormone responsive (THRSP) promotes the synthesis of medium-chain fatty acids in goat mammary epithelial cells. J. Dairy Sci. 99:3124-3133.

Zhou, J., M. Febbraio, T. Wada, Y. Zhai, R. Kuruba, J. He, J. H Lee, S. Khadem, S. Ren, S. Li, R. L. Silverstein, and W. Xie. 2008. Hepatic fatty acid transporter Cd36 is a common target of LXR, PXR, and PPAR $\gamma$ in promoting steatosis. Gastroenterology 134:556-567. 